\title{
La salud como resultado del consumo
}

\section{Consumption-derived health}

\section{Nery Suárez Lugo}

Doctor en Ciencias de la Salud. Investigador Titular. Escuela Nacional de Salud Pública. La Habana, Cuba.

\section{RESUMEN}

Se analizan diversos aspectos que ponen de manifiesto la relación que existe entre el consumo y la salud. Se valoran elementos referentes a la pobreza y la importancia que tiene para la salud pública dada la conexión que existe entre el ingreso individual y familiar y las posibilidades de consumir. Se ejemplifica la responsabilidad del Estado y de las autoridades sanitarias en la oferta de productos seguros y se pone de manifiesto la importancia de la corresponsabilidad entre productor, proveedor y consumidor, que generalmente permite obtener mejores resultados en este asunto. Se destaca el papel de la información y la educación en la toma de conciencia de los consumidores para que ejerzan su función desde la sociedad civil. Se hace énfasis en las consecuencias de los actos de consumo así como su impacto en el bienestar físico, psíquico y social y en la salud pública, lo que expresa la necesidad de políticas que favorezcan la prevención y la promoción de la salud. El desarrollo tecnológico, sobre todo en la esfera de los alimentos procesados, crea incertidumbre y desconfianza en el consumidor, pero la industria se concilia con estos avances y hace un Ilamado para que las políticas sanitarias logren una adecuada relación entre el consumo y la salud.

Palabras clave: Consumo, salud.

\section{ABSTRACT}

Several aspects showing the association of consumption and health were analyzed. Elements linked to poverty and their importance for public health because of the association of individual and family incomes with the consumption possibilities were 
assessed. The responsibility of the state and of the health authorities in the provision of safe products was exemplified; also, the significance of the coresponsibility of producer, provider and consumer that generally leads to better results in this field was set forth. The role of information and education in the consumer's awareness so that they can perform their function in the society was stressed. Emphasis was made on the consequences of the consumption acts as well as the impact on physical, psychical and social welfare and on the public health as well. The technological development, mainly in the field of processed foodstuffs, gives rise to uncertainty and lack of confidence on the part of the consumer; however, the industry reconciles with these advances and calls upon the health policies to reach adequate balance between consumption and health.

Key words: Consumption, health.

\section{SALUD, POBREZA Y POLÍTICAS SANITARIAS}

Salud y pobreza son dos categorías estrechamente relacionadas y todos los que estudian y trabajan en la salud pública tienen total conocimiento y certeza de ello. Sin embargo, se olvida con frecuencia que el consumo es una categoría que define la pobreza, pues precisamente la línea divisoria está trazada por los ingresos de las personas y familias y las posibilidades que este ingreso les ofrece para enfrentarse al mercado y poder acceder o no a los bienes y servicios que requieren para la vida. ${ }^{1}$

Las condiciones de vida se definen por las posibilidades y forma de acceder a los bienes de consumo y los servicios. El estilo de vida no es más que el comportamiento que se tiene ante ellos, marcando la diferencia que este último no está solo determinado por las posibilidades de acceso, sino por las creencias, hábitos y costumbres de los individuos y de los grupos que conforman una determinada sociedad.

A todas luces resulta cierta la relación del consumo con la salud y se puede afirmar que según consumen las personas así será, en última instancia su salud, por lo que si se analiza el comportamiento en el consumo de una población se encontrará explicación a la situación epidemiológica existente.

No obstante, cuando se valoran en la vida diaria los indicadores de salud, generalmente se busca como solucionar los problemas a partir de los servicios sanitarios a ofrecer y cómo y con qué curar a los enfermos. Así se observa como a diario en el discurso se enuncian los siempre llamados "nuevos paradigmas" aunque desde hace más de tres décadas se habla de ellos, sin embargo, la realidad no presenta un enfoque eminentemente preventivo, sino realmente más dirigido a lo asistencial y curativo.

Para lograr comportamientos en el consumo que tributen al bienestar, como concepto actual de salud, en muchas ocasiones hay que transgredir intereses económicos de trasnacionales y a veces de gobiernos y para nadie es un secreto lo difícil que ello resulta. En situaciones como las de Cuba, puede ser más sencillo, sin embargo, todo parece indicar que no se coloca en la agenda de los que deciden las 
políticas públicas, los argumentos que establecen la relación apropiada entre la forma de consumir y su resultante en la salud.

Analizada este problema desde el punto de vista teórico, es necesario considerar el proceso de construcción de tres conceptos fundamentales: cultura, toma de conciencia y sensibilización, que pueden facilitar en unos países formas, contenidos y medios para la mejoría o erradicación de la pobreza, en la medida en que contribuyen a propiciar la racionalidad en el consumo y en otros, como el caso de Cuba, impedir que los cambios en la situación de mercado afecten las conquistas logradas. ${ }^{2}$

Entonces resulta necesario ver la salud desde una perspectiva diferente y para la cual una parte importante de los profesionales sanitarios no se encuentran suficientemente preparados, ya que implica analizar la salud como resultado y consecuencia de los hábitos de consumo de las personas.

Para la construcción a escala poblacional de esos tres conceptos que fueron señalados anteriormente, hay que actuar primero en los trabajadores sanitarios en todos los niveles, desde los que deciden las políticas sanitarias, hasta los encargados de ejecutarlas, para que incorporen al análisis de la situación de salud, la perspectiva del consumo y el comportamiento de la población ante él.

Si bien en lo conceptual el modelo de salud pública ha ido transformándose y aparejado a ello el concepto de salud, los profesionales sanitarios no están lo suficientemente preparados para actuar en ese medio externo y dar respuesta a los nuevos requerimientos de salud. Hay nuevas competencias para el gerente y el profesional sanitario que requieren de una preparación acorde a estas, para darle respuesta en un adecuado desempeño. ${ }^{3}$

\section{EL CONSUMIDOR Y LA OFERTA DE PRODUCTOS SEGUROS}

Uno de los más claros ejemplos del impacto de lo que se consume en la salud es la alimentación de las personas de todos los grupos de edades y principalmente los niños, por la importancia que tiene en el desarrollo físico y psíquico de las personas en todo su ciclo vital.

El Estado tiene la obligación de poner en el mercado productos seguros, a la vez que educar al consumidor en la compra y uso responsable de todos los bienes, tanto de los alimentos, equipos electrodomésticos, como de juguetes y videojuegos, entre otros. No obstante, en gran parte de las ocasiones, es la sociedad civil la que toma las riendas para educar al consumidor y a la vez exigir al Estado la protección legal de sus derechos.

La seguridad de un producto está dada, porque en condiciones de utilización normales o razonablemente previsibles, incluidas las condiciones de duración y, si procede, de puesta en servicio, instalación y mantenimiento, no presente riesgos o solo riesgos mínimos compatibles con el uso del producto y considerados admisibles dentro del respeto de un nivel elevado de protección de la salud y la seguridad de las personas. Debe tener reflejado en su etiqueta las características, instrucciones, advertencias de efectos y riesgos, fecha de caducidad, por citar los elementos más importantes. 
Una nota de prensa publicada por la agencia Reutter, señalala situación confrontada por los europeos dada las cremas solares que se ofertan en el mercado y que prometen protección total, lo que está científicamente demostrado que no es posible. Sin embargo, lo establecido por las autoridades sanitarias de la Unión Europea con la industria desde el año 2006, es que los envases de estas cremas protectoras indiquen lo que es real y no prometer el $100 \%$ de protección, porque no resulta posible y por tanto no es cierto.

Informes de la Organización Mundial de la Salud indican que gran número de personas enferman cada año con cáncer de piel y no se puede pensar solo en los vacacionistas que se exponen al sol, sino en todas aquellas personas que trabajan expuestos durante muchas horas para ganar el sustento suyo y de su familia, como por ejemplo los obreros agrícolas. Lo importante es, que las autoridades sanitarias orienten a la población sobre los peligros a que conlleva la exposición al sol en los horarios entre las 11:00 y las 15:00 h, y como protegerse y no dejar que se conviertan en orientadores los que con fines de lucro producen protectores solares (Reuters Toronto, marzo 17/2008 Diaria. Boletín 18 de marzo de 2008. Infomed).

Otro punto de importancia es que toda la información relacionada tiene que estar el idioma que se hable en el país de referencia. No obstante, hay que considerar también que aún así, los analfabetos, indígenas que hablan otras lenguas y los inmigrantes, son poblaciones excluidas, ya que no tienen acceso a esta información y quedan por tanto desprotegidos.

Sin embargo, es usual encontrar que los consumidores no están pendientes de la seguridad como categoría fundamental para decidir la compra de un producto.

La obligación de poner en el mercado productos seguros es de las empresas, mientras que las administraciones públicas deben velar porque así sea. Por su parte, los consumidores tienen un papel fundamental en la denuncia de artículos que puedan causar o hayan provocado algún tipo de accidente, de manera que las autoridades sanitarias puedan actuar y evitar posibles daños a otras personas. De esta forma se establece una corresponsabilidad entre productor, proveedor y consumidor, que generalmente permite obtener mejores resultados, sin obviar la supervisión de los encargados de velar por la salud pública.

Si bien el problema del consumo no constituye una responsabilidad única de la salud pública, si lo es en todo aquello que implique riesgos para la salud, por lo que les compete propiciar la acción conjunta y coordinada de todas las instancias que pueden hacer posible el proceso de toma de conciencia de la población sobre los riesgos que entraña determinados consumos y a la vez, exigir a los productores y comercializadores la información veraz y fidedigna sobre sus ofertas.

Los consumidores están en mejor situación para juzgar sus propias necesidades y preferencias y para ejercer sus propias opciones cuando son educados desde la niñez para tener un comportamiento racional ante el consumo, que significa tomar decisiones ante las opciones de que dispone de la manera más adecuada.

\section{DISPONIBILIDAD, INFORMACIÓN Y OPORTUNIDADES PARA CONSUMIR. EL DILEMA DEL INGRESO}

La información es entonces el elemento fundamental para dar a conocer los productos y servicios que se ofertan en el mercado y poner a disposición del 
consumidor la diversidad de opciones de consumo disponibles para que decida cuáles son mejores, esto es, cuales satisfacen sus necesidades y no afectan su salud.

Las campañas de publicidad y de información pública se encargan de ello, las primeras, si siguen normas y restricciones establecidas en general en todos los países, son transparentes y ofrecen información veraz y fidedigna, pues contribuyen a que el consumidor tome decisiones acertadas. Al disponer además de los elementos que ofrecen las segundas, pues se logra el equilibrio necesario para propiciar un consumo racional.

También las opciones están en dependencia de la situación económica de cada individuo y familia y las posibilidades de acceso a los bienes y servicios que se requieren para la vida. Hay muchos factores que causan esas restricciones de las opciones de consumo, no siendo el ingreso el único. Disponibilidad, infraestructura, información, así como barreras sociales y culturales, constituyen también factores que condicionan el comportamiento.

Entre las opciones de consumo, la disponibilidad de bienes y servicios, puede valorarse en cuanto a diversidad, pero también desde la perspectiva de la equidad en relación con el papel del Estado como benefactor, al privilegiar los productos que tienen un efecto positivo para la salud, el bienestar y el desarrollo humano.

El ingreso, las opciones disponibles, el tiempo de que se dispone, la información y el hogar, constituyen entonces elementos de importancia para las decisiones que los individuos y los grupos deben tomar en relación con el consumo. Pero cada uno de ellos no puede verse en una correspondencia directa, sino que todos en estrecha relación, provocan de conjunto la conducta individual y social.

El ingreso es un medio importante de aumentar la diversidad de opciones de consumo, pues determina la capacidad de compra, por consiguiente los cambios de ingreso tienen gran influencia sobre las modificaciones del consumo, aunque no necesariamente conduce a mejorar la calidad de vida, motivado por las distorsiones que pueden producirse derivado de una actitud consumista y un consumo irracional. $^{4}$

Las opciones de consumo dependen de la disponibilidad de diversos bienes y servicios, desde el mercado y el aprovisionamiento estatal hasta la producción doméstica y los recursos comunes. Muchos de los bienes y servicios esenciales: agua, saneamiento, educación, atención de salud, transporte y electricidad, no pueden suministrarse sin una infraestructura, lo que constituye una responsabilidad del Estado hacerlos disponibles bajo el concepto de equidad, para que todos tengan acceso a esos servicios. Cuando coexisten servicios públicos y privados debe mantenerse un equilibrio entre ellos para que no provoquen desigualdad social.

Las oportunidades para consumir pueden verse gravemente limitadas por la falta de tiempo. En el mundo industrializado, los estilos de vida excesivamente ocupados, impiden disfrutar de actividades de recreación, pese al elevado ingreso, mientras que en los países pobres, la sobrecarga de trabajo derivado del poco acceso a servicios básicos, hace que la población y fundamentalmente sus mujeres, se vean limitadas en el tiempo. Cuando se sitúa al interior de los países, la situación resulta similar para ricos y pobres y ambas atentan a la calidad de vida, los pobres no pueden acceder y los ricos distorsionan la forma de empleo de sus ingresos.

La información parece ser la clave para aumentar la conciencia de la diversidad de opciones de consumo disponibles y permitir que el consumidor decida cuáles son 
mejores. Sin información no hay manera de saber de los bienes y servicios que existen en el mercado, y qué servicios está prestando el Estado y están a disposición de todos por derecho propio. Las campañas de información pública desempeñan un papel importante a este respecto para hacer que los consumidores tengan conciencia, tanto de los beneficios como de las posibles consecuencias negativas de las opciones que enfrentan.

Los valores domésticos tienen mayor efecto sobre las opciones de consumo de los miembros considerados individualmente. La educación y la crianza que se da a los niños en las primeras etapas de la infancia, desempeñan un papel fundamental en determinar su capacidad para utilizar bien las opciones que tienen para vivir una vida plena y satisfactoria. La notable ampliación y diversificación de las opciones de consumo ha hecho que sea más difícil que los consumidores decidan sobre ellas de manera informada.

Las personas a veces no tienen conciencia de las consecuencias de sus decisiones. Si no se alimenta adecuadamente a un niño pequeño, si no se envía un niño a la escuela, si no se hace que un adolescente conozca la importancia de la atención a la salud reproductiva, si no se da a un joven la oportunidad de desarrollar un sentido de comunidad, no tendrán la misma capacidad que otros para ejercer opciones que eleven a un máximo sus mejores intereses así como de los que conviven con él.

Pero el conocimiento no lo es todo. Un estudio reciente, muestra cómo la salud de una persona puede estar íntimamente relacionada con los factores socieconómicos, como la educación y los índices de ingreso. Investigadores de la Sociedad Estadounidense del Cáncer y de la Universidad de Emory en Atlanta, calcularon las tasas de mortalidad por cáncer de pulmón, mama, próstata y colon, por niveles educativos en personas de raza blanca y negra entre los 25 a 64 años de edad, desde 1993 al 2001 en Estados Unidos.

Las tasas de mortalidad de estos tipos de cáncer disminuyeron en la fecha estudiada en hombres y mujeres con educación universitaria, excepto en el cáncer de pulmón entre las mujeres negras, para quienes la mortalidad se mantuvo estable.

Las reducciones recientes importantes en las tasas de mortalidad por cánceres en Estados Unidos, no incluyen en igual forma a personas menos instruidas en edad laboral, lo que sugiere que quienes tienen menor nivel socioeconómico no se beneficiarían igual con los avances recientes en la prevención, detección temprana y tratamiento oportuno de cánceres más letales.

Los adultos con menos educación son más propensos a fumar, y en el caso del cáncer de mama, las mujeres tienden menos a realizarse mamografía para detectar en estadio temprano la enfermedad. Las personas con menos nivel educativo se someten menos a controles del cáncer colorrectal y a recibir las mejores alternativas de tratamiento para los tumores. Los índices de ingreso se relacionan con los niveles educativos. Las personas con bajos ingresos son menos propensas a contar con una cobertura de salud que aquellas con más recursos financieros (Journal of the National Cancer Institute. Disponible en: http://jncicancerspectrum.oxfordjournals.org/).

El nivel educativo y los ingresos determinan fundamentalmente la elección y los comportamientos alimentarios que, en última instancia, pueden producir enfermedades relacionadas con la dieta. Los orígenes de muchos de los problemas que afrontan las personas con ingresos bajos resaltan la necesidad de un enfoque 
multidisciplinar para encarar las necesidades sociales y atenuar las desigualdades en materia de salud.

El ingreso se comporta como una variable trazadora, que permite identificar el comportamiento de los individuos en dependencia de su nivel educacional, el acceso a la información y la disponibilidad de las opciones y oportunidades para consumir. Conociendo el ingreso de las personas de manera individual y familiar, se puede conocer y por tanto pronosticar, con una probabilidad bastante certera, cual será su comportamiento en el consumo y derivado de ello su estado de bienestar, constituyendo por tanto una información de gran valor para los que se ocupan de la salud pública.

\section{CONSECUENCIAS DE LOS ACTOS DE CONSUMO}

El consumo es consecuencia de diversos factores, muchos de ellos externos al consumidor pero que influyen y en algunos casos determinan, su comportamiento.

Por ejemplo, la incorporación creciente de los ciudadanos al proceso de globalización y "modernización" ha provocado cambios en el campo del desarrollo individual e interpersonal, donde el avance tecnológico contrasta con el empobrecimiento de los recursos de las personas para expresarse emocional y socialmente, disminuyendo su calidad de vida y afectando el bienestar psicosocial. ${ }^{5}$ Analizando el comportamiento en el consumo de una población, se encuentran las explicaciones a cómo y de qué enferman y mueren.

A la vez, el consumo tiene consecuencias que no solo afectan al propio consumidor, pues aunque los individuos adoptan las decisiones relativas al consumo que tienen una connotación individual, también tienen efectos sobre otros, a escala individual o social, e inclusive, pueden tener vínculos con el desarrollo humano.

El adquirir un determinado producto u obtener un servicio, tiene consecuencias directas para el consumidor, al que le puede proporcionar satisfacción, si responde totalmente a sus expectativas o frustración si no resulta de esta forma. Tanto una como la otra están relacionadas, de manera positiva o negativa, con el estado de bienestar del individuo.

También tiene consecuencias para la sociedad, pues la forma en que un individuo se comporta en el consumo, es una expresión del grupo social, las normas y patentes culturales al que se quiere pertenecer. Es por consiguiente una forma de encontrar explicaciones a situaciones sociales que pueden presentarse, pues por ejemplo, cuando se encuentra que la población prefiere adquirir determinados artículos, como puede ser la ropa, siguiendo patrones alejados de su cultura, está perdiendo sus raíces y ello puede observarse desde la música que se consume hasta los alimentos.

Es una forma de desplazamiento a adquirir los bienes y servicios por el valor de signo y no por el valor de cambio y de uso, pero un signo que se forma a partir de un proceso de transculturación de la sociedad que generalmente, aunque puede valorarse como desarrollo, también trae consecuencias negativas para la sociedad. ${ }^{6}$

El consumo tiene a su vez consecuencias ambientales que están relacionadas con la contaminación de las aguas por los desechos que se vierten de manera inadecuada, la contaminación de los cultivos por la toxicidad de algunos pesticidas y plaguicidas 
y también la explotación indiscriminada de los recursos naturales que atentan con la necesidad de un desarrollo sostenible para no poner en peligro la vida en el planeta. Está comprobada la acción de los aerosoles en el daño de la capa de ozono y sin embargo se siguen produciendo porque los consumidores los siguen adquiriendo. Tal importancia tiene, que el Protocolo de Kyoto ha sido una necesidad de la humanidad para poner sobre los hombros de los gobiernos esta responsabilidad.

Hay consumos que sus consecuencias están ligadas de manera muy clara a la salud, la calidad de vida y la mortalidad inclusive, tal es el caso del consumo de cigarrillos y otros productos manufacturados del tabaco, el consumo excesivo de alcohol, la alimentación inadecuada y por supuesto, las drogas ilícitas.

La vacunación contra una enfermedad contagiosa reduce los riesgos de salud, tanto del que se vacuna como de otros, pero sin embargo, cuando una persona fuma en presencia de terceros, los está convirtiendo en fumadores pasivos, cuando ingiere bebidas alcohólicas en exceso daña a su familia y hasta la comunidad. Todas ellas, de manera positiva o negativa tienen además efectos externos.

Los actos de consumo en el orden individual, permiten diagnosticar el estado de salud de una persona; si se trata de un grupo, comunidad e inclusive nación, también resulta factible diagnosticar el estado de bienestar físico, psíquico y social, por consiguiente, estudiar los hábitos de consumo, resulta una responsabilidad de la salud pública, no sólo para resolver los problemas del presente, sino para ejercer una acción prospectiva que permita en el futuro mejorar el estado de salud de la población. ${ }^{7}$

El consumo opera como potente mecanismo de integración social y participar en el consumo de bienes y servicios deviene en una de las modalidades principales para participar en el proceso social, lo que hace que tenga su efecto en los individuos y las familias que asumen una actitud no racional y consumista y quieran apropiarse de productos y servicios solo porque ello tiene un efecto significativo en los procesos de reconocimiento y de pertenencia a determinados grupos sociales que realmente se convierten en referencia, pero que el nivel de ingreso no permite que sean de pertenencia.

Precisamente ese significado del consumo genera inseguridad y puede provocar hasta el endeudamiento de personas y familias con sus consecuencias en la salud psíquica de sus integrantes. Las ventas a crédito, los préstamos bancarios y las hipotecas, resuelven problemas del presente pero en una gran mayoría generan problemas a largo plazo para el individuo, la familia y la sociedad.

\section{LA CIENCIA, LA INCERTIDUMBRE Y LA DESCONFIANZA EN EL CONSUMIDOR}

El avance de la investigación científica conlleva también a descubrimientos relacionados con los diferentes productos que se consumen y su relación positiva o no con la salud. Tal es así, que lo que ayer era considerado como beneficioso o al menos neutro, hoy se ha convertido en dañino y aunque la educación de las personas también progrese, queda un poco de incertidumbre, a veces desconfianza y hasta desconcierto, en relación con qué y cómo consumir. 
Hay productos como el tabaco cuyo efecto desde tiempos remotos ha sido conocido como dañino, pero si se analiza el caso del alcohol, se presentan los efectos negativos unido a los positivos de algunas bebidas en determinadas cantidades y cada cual lo interpreta de la manera que considera o le resulte más conveniente.

También esto trasciende a los medicamentos y otros productos farmacéuticos con consecuencias negativas para la salud luego de estudios realizados sobre sus efectos adversos.

Un ejemplo reciente es el relacionado con los suplementos antioxidantes. Hasta el momento se describen sus bondades en estudios realizados en alimentos con propiedades antioxidantes y no con antioxidantes sintéticos, en los que se indican que los nutrientes con estas propiedades pueden resultar beneficiosos contra el cáncer o las enfermedades cardiovasculares. La base teórica se sustenta en que en muchos de estos trastornos las células sufren procesos oxidativos, y como al parecer los antioxidantes eliminan sustancias que favorecen ese daño celular, los denominados radicales libres podían frenar el proceso.

Para comprobar lo anterior, hace unos años los investigadores daneses, conducidos por Goran Bjelakovic, analizan los efectos de los antioxidantes en el riesgo de cáncer de estómago. Entonces, no sólo se encuentran con que los suplementos no protegían de este tumor, sino que las personas que los tomaban parecían tener una mayor mortalidad. El hallazgo les llevó a emprender la investigación tomando como base todos los ensayos clínicos que se habían realizado con suplementos de antioxidantes. En total, encuentran 68 ensayos realizados en más de 232600 personas, tanto en voluntarios sanos, para ver si conseguían prevenir enfermedades, como en pacientes con diversas afecciones, para ver si se evitaba que se agravasen sus dolencias.

El análisis inicial no mostró diferencias en la mortalidad entre los que tomaban antioxidantes y quienes no. Sin embargo, cuando los autores descartaron 21 ensayos de dudosa calidad, salieron a la luz los riesgos de estos suplementos, Por ejemplo, el betacaroteno, la vitamina A y la vitamina $\mathrm{E}$, solos o en combinación con otros antioxidantes, parecen aumentar el riesgo de muerte en el 7, 16 y $4 \%$, respectivamente.

Al considerar globalmente todos los antioxidantes, el riesgo de muerte entre quienes lo consumían era $5 \%$ superior al de las personas que no tomaban estos nutrientes. La vitamina $C$, por el contrario, no parece tener riesgos para la salud. El selenio, por su parte, podría resultar beneficioso, pero los investigadores son prudentes y dicen que hacen falta más estudios antes de concluir que este nutriente prolongue la vida.

Los resultados obtenidos solo son aplicables a los antioxidantes sintéticos, no así a los que se obtienen de los alimentos. Esto es, frutas y verduras, cuyos efectos no han sido estudiados de igual forma y pudieran ser diferentes, ya que cuando se administran a modo de suplementos, la ingesta de antioxidantes es mucho mayor que la obtenida a través de la dieta.

Existen otras alternativas y posibles explicaciones a estos hallazgos, que pueden explicarse mediante el análisis de la propia enfermedad. ${ }^{8}$

Las lecciones aprendidas, desde la perspectiva del consumo y su relación con la salud, pueden ser dos. La primera, que siempre es preferible la opción de encontrar en los alimentos una forma de preservar y mejorar la salud; la segunda, que la ciencia encuentra cada día explicación a muchos fenómenos y hay que estar al 
tanto de lo que va sucediendo para orientar, de manera adecuada, tanto a los profesionales sanitarios como a la población.

\section{ALIMENTOS GENÉTICAMENTE MODIFICADOS Y NANOALIMENTOS, MÁS TEMORES PARA LOS CONSUMIDORES}

La introducción en el mercado de los alimentos genéticamente modificados o aquellos provenientes de animales clonados, se une hoy a la presencia de los denominados nanoalimentos que están entrando silenciosamente en el mercado.

La nanotecnología involucra el diseño y la manipulación de materiales a escala molecular, es decir con menos grosor que un cabello e invisible a simple vista para el ojo humano.

Las compañías que utilizan estas técnicas afirman que pueden realzar el sabor o sus efectos nutricionales. Esta tecnología se basa en efectos cuánticos que hacen que las propiedades de la materia cambien a escala nanométrica.

La investigación sobre las consecuencias a la salud del consumo de este tipo de alimentos producto de la ingeniería genética y sus grandes avances, queda aún por hacer, por lo que se vuelve riesgosa su presencia en el mercado y más aún sin la debida advertencia. Estudios recientes han demostrado que las partículas nanométricas pueden invadir las células en algunos casos y que ciertas formas de carbono de este tipo pueden ser tan nocivas como el asbesto.

Los nuevos productos creados a través de la Nanotecnología entran al mercado estadounidense y europeo a un ritmo de tres o cuatro por semana y no existen requerimientos para que estos productos sean identificados. Los nanoproductos de uso común en la actualidad incluyen raquetas de tenis, bicicletas, pantallas solares y alimentos como batidos dietéticos, aceites y té.

Una información publicada por la agencia de noticias Reuters en julio de 2008 refiere que hay encuestas que muestran que $69 \%$ de los estadounidenses están preocupados por el consumo de carne de animales clonados.

Los defensores de los consumidores en la sociedad civil estadounidense han pedido a las autoridades sanitarias que se refuercen las regulaciones para confirmar la seguridad

en pruebas y la información en las etiquetas, pero realmente, si bien es importante que los consumidores jueguen su papel, la primacía en cuanto a garantizar la seguridad de los productos que se consumen tiene que ser responsabilidad del Estado y las instituciones sanitarias abogar por ello.

La información en las etiquetas es algo bien importante, pero la complejidad del análisis que se requiere realizar hoy en día demanda de un mayor conocimiento por parte del consumidor, que implica que tenga un nivel educacional acorde a los datos que necesita procesar. Campañas educativas acerca del empleo de tecnologías sofisticadas como las que se describen, permiten transparencia y a la vez, que los consumidores puedan tomar decisiones acertadas en relación con los productos que van a adquirir.

La ciencia y el desarrollo van de la mano, pero es importante que se tenga presente como, en determinadas ocasiones, puede afectar la salud de las personas. La 
investigación científica debe acompañar la introducción de los productos en el mercado, el requerido ensayo clínico en el caso de los medicamentos constituye una alternativa a pensar, cada vez más, si se quiere que los productos que las personas consuman, ya sea alimentos, cosméticos e incluso otros, tengan impacto en el bienestar de la humanidad.

\section{LA INDUSTRIA Y SU CONCILIACIÓN CON LA CIENCIA}

Los descubrimientos de la ciencia llegan a todas las esferas, siendo la de los alimentos procesados de manera industrial uno de las más importantes. Las instancias sanitarias promocionan un estilo de vida donde no prevalezca el colesterol y la obesidad y las personas buscan opciones más saludables de consumir. ${ }^{9}$

Es conocido que el posicionamiento de los productos los determina, en última instancia, el consumidor, quien al dejar de consumir un determinado producto, les quita participación en el mercado. ${ }^{10}$ Prueba evidente de ello es como las gaseosas tradicionales, en dos años perdieron $4,4 \%$ de participación de ventas al tiempo que creció lo light, lo saborizado y lo energético. En el 2004 del total de ventas de bebidas sin alcohol las gaseosas "regulares" o tradicionales representaban 35,6\% al tiempo que en 2006 este segmento sumó 31,3\% del rubro. En el mismo período, la venta de los jugos en polvo reducidos en calorías se incrementó $2,1 \%$, las aguas saborizadas crecieron $1,9 \%$ y las aguas minerales crecieron $0,8 \%$. Por su parte, las gaseosas light crecieron 0,3\%.

Muchas marcas tradicionales debieron adaptarse al nuevo modelo del consumidor. A veces lo logran con cambios efectivos en la oferta de servicios o productos, como el menú en tono light y saludable que hoy oferta Mc Donald's, y otras veces a través de la extensión de marcas o bien por vía de un cambio de imagen.

Es positiva la tendencia de ofertar productos saludables, las cifras lo certifican en un rubro tan sensible como el de las gaseosas. Todo lo que contiene azúcar, parece estar decreciendo mientras que el consumo de todo lo que es reducido en calorías, energizado o saborizado está incrementándose.

No es casual entonces que una marca como Coca Cola, el emblema de las gaseosas, intentara cambiar de imagen. Según documenta Advertising Age, la gaseosa trata de ser considerada una sparkling water. El vocablo sparkling, se traduce como chispeante, centelleante o espumoso. De este modo la gaseosa pasaría a tener connotaciones buenas ya que el apodo connota simpatía.

Como ahora lo carbonatado cambió su significado, las grandes marcas prefieren escaparle a la categoría de carbonated soft drink. A su vez, en lugar de referirse a bebidas no carbonatadas también prefieren hablar de still, o sea, las bebidas sin gas. De algún modo se trata de un eufemismo, se sigue hablando de lo mismo pero es conveniente no usar vocablos que tal vez despierten desconfianza como gaseosa o carbonatada. Son varias las marcas de gaseosas que salieron a buscar mercado al poner en primer plano que eran "finamente gasificadas". Pero una marca como Coca Cola, en su versión clásica, no puede eludir la presencia de gas, es más, los consumidores rechazan la falta de gas porque así se arruina el buscado y esperado efecto burbujeante. 
Como ya ha sido analizado, hay convicción de que la industria sigue muy de cerca lo que la ciencia demuestra, y en ocasiones son ellos los que hacen que el consumidor cambie su manera de consumir. La industria se adapta a los cambios de consumo, a veces antes que ocurran y readapta sus discursos sin perder identidad, pues es eso lo que lo identifica en el mercado y está posicionado en la mente del consumidor.

Las lecciones aprendidas en relación con los productores, se refieren a que su motivo principal es la ganancia, se produce para vender. Es por eso que los productores se mantienen muy al tanto de lo que sucede en el ámbito científico de todos aquellos productos que tienen relación con la salud.

Ante las intensivas campañas sobre la nocividad del cigarrillo y que las personas dejen de fumar, reducen los niveles de nicotina y alquitrán, crean cigarrillos falsamente denominados light, mientras que aparecen toda una gama de productos dirigidos a reemplazar la nicotina y ayudar a los fumadores a reducir y eliminar su adicción.

La industria alimenticia, se coloca a la cabeza, en la búsqueda de alternativas, que van desde los aceites y todos sus derivados que se anuncian como carentes de colesterol, al ejemplo de las gaseosas que se analizó de manera particular. ${ }^{11}$

A su manera, y en su intención de mantener las ganancias, la industria se convierte en aliado de la institución sanitaria para promover consumos saludables. Queda entonces que realmente sea así y la salud pública se verá beneficiada.

\section{REFERENCIAS BIBLIOGRÁFICAS}

1. Fafard P. Evidence and Healthy Public Policy: insights from Health and Political Sciences National. Quebec: Collaborating Centre for Healthy Public Policy; 2008.

2. Bruner J. La educación, puerta de la cultura. Madrid: Editorial Visor; 2001.

3. Suárez Lugo N. Mercadotecnia en el Sistema Nacional de Salud de Cuba. 2005. Rev Cubana Salud Pública. 2006;34(1):1-11.

4. Irala-Estevez J, Groth M, Johansson L, Oltersdorf U, Prattala R, MartinezGonzalez MA. A systematic review of socioeconomic differences in food habits in Europe: consumption of fruit and vegetables. European J Clin Nut. 2000;54:70614.

5. Rey G. Cultura y Desarrollo Humano: Unas relaciones que se trasladan. Colombia: El Espectador; 2002.

6. Suárez Lugo N. Enfoque Social de la Mercadotecnia Sanitaria. La Habana: Editorial Ciencias Médicas; 2007.

7. Nilsen $P$, Bourne $M$, Verplanken $B$. Accounting for the role of habit in behavioural strategies for injury prevention. Int J Inj Contr Saf Promot. 2008;15(1):33-40.

8. Discovery dsalud ¿Son o no eficaces los antioxidantes? [sitio en Internet]. [citado 5 Ago 2008]. Disponible en: http://www.forosperu.net/showthread.php?t=26536\&page $=9$ 
9. Angeleri MA, González I, Ghioldi MM, Petrelli L. Hábito de consumo del desayuno y calidad nutricional del mismo en niños y adolescentes de la zona norte del Gran Buenos Aires. Dieta. 2007;25(119):7-13.

10. Suárez Lugo N. Marketing en Salud: una perspectiva teórico-práctica. Cali (Col): Editorial CATORSE;2004.

11. Dibsdall LA, Lambert N, Bobbin RF, Frewer LJ. Low-income consumers' attitudes and behaviour towards access, availability and motivation to eat fruit and vegetables. Public Health Nutr. 2003;6(2):159-68.

Recibido: 13 de marzo de 2009.

Aprobado: 2 de mayo de 2009.

Nery Suárez Lugo. Escuela Nacional de Salud Pública. Calle Línea esq. a I. El Vedado 10400. La Habana, Cuba.

E-mail: nerysl@infomed.sld.cu 